\title{
Prevalence of pulmonary embolism in syncope patients
}

\author{
Sean Crooks, BSc*; Eddy Lang, $\mathrm{MD}^{\dagger}$
}

Clinical question

How often is pulmonary embolism (PE) found in patients admitted for syncope?

Article chosen

Prandoni $\mathrm{P}$, Lensing $\mathrm{A}$, Prins $\mathrm{M}$, et al. Prevalence of pulmonary embolism among patients hospitalized for syncope (PESIT). N Engl J Med 2016;375:1524-31, doi: 10.1056/NEJMoa1602172.

Objective

To determine the prevalence of PE in patients hospitalized for a first episode of syncope.

Keywords: pulmonary embolism, syncope, venous

thromboembolism

\section{BACKGROUND}

Syncope is defined as an abrupt and transient loss of consciousness with complete and sudden spontaneous recovery. ${ }^{1}$ Episodes of syncope are a common reason for presentation to emergency departments (EDs), comprising up to $3 \%$ of visits. ${ }^{2}$ The differential diagnosis for the etiology of syncope is wide and consists of neurologic, volume depletion, and cardiovascular causes such as pulmonary embolism (PE). ${ }^{1}$ Due to more frequent etiologies for syncope, $\mathrm{PE}$ is considered to be an uncommon cause. ${ }^{1}$

Although $\mathrm{PE}$ is not a common cause of syncope, a syncopal episode can be an important symptom of PE. 3,4 Syncope as an initial presentation of $\mathrm{PE}$ has been reported to be as high as $10 \% .^{5,6}$ However, accurate diagnosis of $\mathrm{PE}$ can be challenging because the most common clinical features - such as dyspnea, shock, syncope, and hemoptysis - have minimal diagnostic value in isolation. ${ }^{4,7}$ The diagnosis of $\mathrm{PE}$ is exceptionally important because it is a common cause of hospital admission and mortality. ${ }^{7,8}$ The Pulmonary Embolism in Syncope Italian Trial (PESIT) reviewed in this article was conducted to determine the prevalence of $\mathrm{PE}$ in patients hospitalized through the ED for syncope.

\section{POPULATION STUDIED}

Patients older than 18 years who presented to the ED and subsequently admitted to the medical ward for their first episode of syncope were enrolled in this study. Any patient with previous episodes of syncope or who received anticoagulants or was pregnant was excluded.

\section{STUDY DESIGN}

PESIT was a multicentre cross-sectional study with the primary objective of determining the frequency of $\mathrm{PE}$ in hospitalized patients. Once admitted to hospital, patients were assessed by physicians trained in the study protocol, according to the 2014 European Society of Cardiology (ESC) Syncope Guidelines and Simplified Wells Score. ${ }^{1,9,10}$ All patients underwent chest radiography, arterial blood gas, and blood testing, including a D-dimer assay.

Patients with a high pretest probability based on the Wells Score and/or a positive D-dimer were sent for a computed tomography pulmonary angiogram (CTPA). If patients had a history of kidney impairment or contrast allergy, ventilation-perfusion (V-Q) scanning was used. An autopsy was used if patients died before imaging. For patients with a low pretest probability and a negative D-dimer, no further testing was done.

From the *Cumming School of Medicine; and the tDepartment of Emergency Medicine, University of Calgary, Calgary, AB.

Correspondence to: Sean Crooks, Health Sciences Centre - Cumming School of Medicine, 3330 Hospital Drive NW, Calgary, AB T2N 4N1; Email: smcrooks@ucalgary.ca 


\section{OUTCOME MEASURED}

The primary outcome was the presence of $\mathrm{PE}$ with an intraluminal filling defect on CTPA or a defect of at least $75 \%$ of a segment with corresponding normal ventilation.

\section{RESULTS}

Patients totalling 2,584 presented to the ED with syncope, of whom 717 were admitted to hospital; 118 patients were excluded due to current anticoagulation therapy for atrial fibrillation or unspecified reasons, 35 for recurrent syncope, and 4 refused consent. This left 560 patients with first episode of syncope to be included in the study. For 330 patients, PE was ruled out based on low pretest probability and a negative $\mathrm{D}$-dimer. Of the remaining 230 , 135 had a positive D-dimer only, 3 patients had a high pretest probability only, and 92 patients had both. PE was confirmed in 97 of the 230 patients. In patients hospitalized with syncope, the prevalence of PE was $17.3 \%$ (95\% confidence interval, 14.2-20.5).

\section{COMMENTARY}

It is important to remember that the $17 \%$ prevalence of $\mathrm{PE}$ in syncope patients reported in PESIT was only for the patients admitted to the hospital and did not include all syncope patients who presented to the ED. From the $\mathrm{ED}, 72 \%$ were discharged with benign causes of syncope such as vasovagal or orthostatic. Patients with $\mathrm{PE}$ represented only $3.7 \%$ of the original ED syncope patients. However, this may underestimate the prevalence of $\mathrm{PE}$ in discharged patients because they were not systematically screened or followed for PE. The total number of syncope patients with PE in the ED in PESIT was still higher than the $1 \%$ prevalence of $\mathrm{PE}$ in syncope patients reported from previous studies. ${ }^{11}$

In the PESIT study, 27\% of syncope patients from the ED were admitted to the hospital. A 27\% admission rate for syncope was appropriate considering the wide variation in rates worldwide. For example, the $27 \%$ in PESIT was double the $13 \%$ in Canada, but less than the elevated $50 \%$ admission rate in the United States and Europe. ${ }^{12-14}$ However, the criteria to admit to the hospital were not standardized, and the decision was made by physicians aware of the goals of the study. The cohort admitted to the hospital had multiple comorbidities such as cardiac disease and cancer and a median age of 80 years. This older and sicker cohort likely may have contributed to a higher estimation of prevalence of $\mathrm{PE}$.

One reassuring finding is that many of the diagnosed PE patients had risk factors and clinical features consistent with $\mathrm{PE}$. For example, $45 \%$ of patients with $\mathrm{PE}$ were tachypneic, 33\% were tachycardic, $40 \%$ had signs of deep vein thrombosis (DVT), and 20\% had active cancer. Consequently, many of these patients would have been considered high risk and would have been identified in the ED using common screening tools. ${ }^{1,9,15,16}$ Screening for $\mathrm{PE}$ in patients with clinical signs of $\mathrm{PE}$ is appropriate; however, the blanket approach used in PESIT, where all syncope patients were screened, is not. This is especially relevant because 135 of the 235 hospitalized patients were imaged with a low pretest probability and a positive D-dimer only. In the case of low pretest probability, the Pulmonary Embolism Rule Out Criteria (PERC) rule should have been used to decrease the use of D-dimer testing and prevent over-imaging. ${ }^{17}$

Over-imaging in $\mathrm{PE}$ also increases the risk of false-positive imaging results. False-positive imaging rates are not insignificant with CTPA having 26\% false-positive rate and V-Q scanning having up to $12 \%$ false-positive rate for high probability scans. ${ }^{18,19}$ These rates may be due to a tendency for radiology to favor a positive result due to the consequences of a falsenegative or "missed" PE. However, false-positive PEs are not benign and expose the patient to the unnecessary cost and bleeding risk of prolonged anticoagulation. Falsely diagnosed patients may also experience the stigma and anxiety associated with a label of PE. ${ }^{20}$ For example, they may no longer qualify for travel insurance and often seek future medical aid for the mildest of symptoms.

Increased imaging with CTPA can also unveil asymptomatic PE or PE of uncertain clinical significance. ${ }^{4,20}$ In PESIT, $25 \%$ of PE patients had no symptoms other than syncope. Other studies have shown that up to $3 \%$ of PEs are asymptomatic. $^{21,22}$ Therefore, many of the PEs may have been pre-existing and not the cause of syncope. From the results, it was impossible to determine the time of onset of the PE and whether PE was the cause of the syncope. In addition, some of the PEs found in PESIT may not have clinically significant or required treatment. For example, $6.9 \%$ of PEs diagnosed with a CT in PESIT were subsegmental, in which anticoagulation treatment is controversial. ${ }^{23}$

PESIT begs the question of whether all patients with syncope require a full workup to rule out $\mathrm{PE}$. 
Systematic screening for PE in syncope patients with laboratory and radiographic investigation would lead to unnecessary testing, risks, and costs. Considering that $\mathrm{PE}$ is an uncommon etiology in ED syncope patients, continuing with the safe, cost-effective manner of using validated screening tools, such as the Wells Score and PERC rule, to assess pretest probability and decide D-Dimer testing is sufficient to rule out PE.,17

\section{CONCLUSION}

The findings of the PESIT trial came as a surprise to many physicians who consider PE too low on the differential diagnosis for syncope. It is important to reiterate that the prevalence of PE in PESIT reflects older and multiple comorbid hospitalized patients and not the ED patient population. The findings may help bring $\mathrm{PE}$ to the front of the minds of ED physicians in the workup of unexplained syncope. This is a positive development considering the seriousness of $\mathrm{PE}$; however, this could lead to increased costs and harm due to less discriminating CTPA use.

Competing interests: None declared.

\section{REFERENCES}

1. Brignole $\mathrm{M}$, Alboni $\mathrm{P}$, Benditt $\mathrm{D}$, et al. Guidelines on management (diagnosis and treatment) of syncope update 2004. Eur Heart 7 2004;25(22):2054-72.

2. Moya A, Sutton R, Ammirati F, et al. Guidelines for the diagnosis and management of syncope (version 2009). Eur Heart 7 2009;30(21):2631-71.

3. Thames M, Alpert J, Dalen J. Syncope in patients with pulmonary embolism. JAMA 1977;238(23):2509-11.

4. West J, Goodacre S, Sampson S. The value of clinical features in the diagnosis of acute pulmonary embolism: systematic review and meta-analysis. Q7M 2007;100(12):763-9.

5. Calvo-Romero J, María J, Pérez-Miranda M, et al. Syncope in acute pulmonary embolism. Eur 7 Emerg Med 2004; 11(4):208-9.

6. Altınsoy B, Erboy F, Tanriverdi H, et al. Syncope as a presentation of acute pulmonary embolism. Ther Clin Risk Manag 2016;12:1023.

7. Castelli R, Tarsia P, Tantardini C, at al. Syncope in patients with pulmonary embolism: comparison between patients with syncope as the presenting symptom of pulmonary embolism and patients with pulmonary embolism without syncope. Vasc Med 2003;8(4):257-61.

8. Wolfe T, Allen T. Syncope as an emergency department presentation of pulmonary embolism. 7 Emerg Med 1998; $16(1): 27-31$.
9. Wells P, Ginsberg J, Anderson D, et al. Use of a clinical model for safe management of patients with suspected pulmonary embolism. Ann Intern Med 1998;129(12): 997-1005.

10. Van Belle A, Buller H, Huisman M, et al. Effectiveness of managing suspected pulmonary embolism using an algorithm combining clinical probability, D-dimer testing, and computed tomography. 7AMA 2006;295(2):172-9.

11. Sarasin F, Louis-Simonet M, Carballo D, et al. Prospective evaluation of patients with syncope: a populationbased study. Am 7 Med 2001;111(3):177-84.

12. Thiruganasambandamoorthy $\mathrm{V}$, Hess E, Alreesi A, et al. External validation of the San Francisco Syncope Rule in the Canadian setting. Ann Emerg Med 2010;55(5):464-72.

13. Elesber A, Decker W, Smars P, et al. Impact of the application of the American College of Emergency Physicians recommendations for the admission of patients with syncope on a retrospectively studied population presenting to the emergency department. Am Heart $\mathcal{F} 2005 ; 149(5)$ : 826-31.

14. Bartoletti A, Fabiani $\mathrm{P}$, Adriani $\mathrm{P}$, et al. Hospital admission of patients referred to the Emergency Department for syncope: a single-hospital prospective study based on the application of the European Society of Cardiology Guidelines on syncope. Eur Heart 7 2006;27(1):83-8.

15. Quinn J, McDermott D, Stiell I, et al. Prospective validation of the San Francisco Syncope Rule to predict patients with serious outcomes. Ann Emerg Med 2006;47(5): 448-54.

16. Carrier M, Wells $\mathrm{P}$, Rodger $M$. Excluding pulmonary embolism at the bedside with low pre-test probability and D-dimer: safety and clinical utility of 4 methods to assign pre-test probability. Thromb Res 2006;117(4):469-74.

17. Kline A, Courtney M, Kabrhel C, et al. Prospective multicenter evaluation of the pulmonary embolism rule out criteria. 7 Thromb Haemost 2008;6(5):772-80.

18. Hutchinson B, Navin P, Maron E, et al. Overdiagnosis of pulmonary embolism by pulmonary CT angiography. $A 7 R$ Am 7 Roentgenol 2015;205(2):271-7.

19. PIOPED Investigators. Value of the ventilation/perfusion scan in acute pulmonary embolism. Results of the prospective investigation of pulmonary embolism diagnosis (PIOPED). FAMA 1990;263(20):2753.

20. Wiener R, Schwartz L, Woloshin S. When a test is too good: how CT pulmonary angiograms find pulmonary emboli that do not need to be found. BM7 2013;347:f3368.

21. Dentali F, Ageno W, Becattini C, et al. Prevalence and clinical history of incidental, asymptomatic pulmonary embolism: a meta-analysis. Thromb Res 2010;125(6): 518-22.

22. Schultz D, Brasel K, Washington L, et al. Incidence of asymptomatic pulmonary embolism in moderately to severely injured trauma patients. 7 Trauma Acute Care Surg 2004;56(4):727-33.

23. Torbicki A, Arnaud P, Konstantinides S, et al. Guidelines on the diagnosis and management of acute pulmonary embolism. Eur Heart 7 2008;29(18):2276-315. 